PAPER

\title{
Risk and protective effects of the APOE gene towards Alzheimer's disease in the Kungsholmen project: variation by age and sex
}

\author{
C Qiu, M Kivipelto, H Agüero-Torres, B Winblad, L Fratiglioni
}

J Neurol Neurosurg Psychiatry 2004;75:828-833. doi: 10.1136/jnnp.2003.021493

See end of article for authors' affiliations

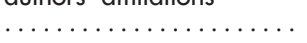

Correspondence to: Dr C Qiu, Stockholm Gerontology Research Centre, Box 6401 (Olivecronas väg 4), S-113 82 Stockholm Sweden; chengxuan.qiu@ neurotec.ki.se

Received 28 June 2003 Revised 9 October 2003 Accepted 12 October 2003
Background: The risk effect of APOE $\varepsilon 4$ allele for Alzheimer's disease is acknowledged, whereas the putative protective effect of $\varepsilon 2$ allele remains in debate.

Objectives: To investigate whether those inconsistent findings may be attributable to differences in age and sex composition of the study populations.

Methods: A community dementia free cohort $(n=985)$ aged $\geqslant 75$ years was followed up to detect Alzheimer's disease cases (DSM-III-R criteria). Data were analysed using Cox models with adjustment for major potential confounders.

Results: Over a median 5.6 year follow up, Alzheimer's disease was diagnosed in 206 subjects. Compared with APOE $83 / \varepsilon 3$ genotype, the relative risk (RR) of Alzheimer's disease was 1.4 (95\% confidence interval $(\mathrm{Cl}), 1.0$ to $2.0 ; \mathrm{p}=0.03)$ for heterozygous $\varepsilon 4$ allele and $3.1(95 \% \mathrm{Cl}, 1.6$ to 5.9$)$ for homozygous $\varepsilon 4$ allele. The association between $\varepsilon 4$ allele and Alzheimer's disease risk was stronger in men than in women ( $R R$ related to the interaction term $\varepsilon 4$ allele by sex, $0.4 ; 95 \% \mathrm{Cl}, 0.2$ to 0.9 ). The $\varepsilon 4$ allele accounted for one third of Alzheimer's disease cases among men, but only one tenth among women. The $\varepsilon 2$ allele was related to a reduced Alzheimer's disease risk mainly in people aged $<85$ years (RR, 0.4; $95 \% \mathrm{Cl}, 0.2$ to 0.8$)$. The RR of Alzheimer's disease related to the interaction term of $\varepsilon 2$ allele by age was $2.4(95 \% \mathrm{Cl}, 1.0$ to $6.0 ; p=0.06)$.

Conclusions: The APOE genotype specific effects on Alzheimer's disease vary by age and sex, in which the $\varepsilon 4$ allele has a stronger risk effect in men, and the $\varepsilon 2$ allele confers a protective effect only in younger-old people.
$\mathrm{T}$ he $\varepsilon 4$ allele of the apolipoprotein E gene (APOE) has been found to be an important genetic risk factor for late onset Alzheimer's disease in a large majority of epidemiological studies. ${ }^{1-3}$ Conversely, the relation between APOE $\varepsilon 2$ allele (mostly the $\varepsilon 2 / \varepsilon 3$ genotype and rarely the $\varepsilon 2 / \varepsilon 2$ genotype) and risk of Alzheimer's disease remains in much controversy. Although the $\varepsilon 2$ allele was found to be a risk factor for early onset Alzheimer's disease, ${ }^{4}$ the protective effect of the allele on Alzheimer's disease in white people had been clearly indicated in a meta-analysis that covered the major studies investigating this topic until 1997..$^{5}$ Lately, a number of population based studies have, however, shown that the $\varepsilon 2$ allele has no protective effect on Alzheimer's disease ${ }^{6-8}$ or was related to a non-significantly decreased risk of the disease $\mathrm{e}^{9-12}$ probably because of few carriers of the allele. Therefore, the suggested benefit of the $\varepsilon 2$ allele on Alzheimer's disease needs to be further elucidated in large scale community based studies.

The variations by age and sex in the APOE $\varepsilon 4$ specific risk for late onset Alzheimer's disease have been a focus of numerous epidemiological studies. The $\varepsilon 4$ allele associated risk for Alzheimer's disease is frequently reported to decline after around 70 years. ${ }^{5}{ }^{13-15}$ In contrast, there has been a lot of debate about whether there is a sex difference in the $\varepsilon 4$ allele related risk for Alzheimer's disease. The population based prospective data from Finland showed that the association between APOE $\varepsilon 4$ and Alzheimer's disease was more pronounced in men than in women, ${ }^{16}{ }^{17}$ which is in disagreement with some previous studies that show no sex difference ${ }^{11}$ or an apparent stronger $\varepsilon 4$ alleleAlzheimer's disease association in women than in men. ${ }^{18}$ By contrast, very few data are currently available concerning the age and sex variations of the $\varepsilon 2$ specific effect on Alzheimer's disease.

On the basis of previous population based studies, we hypothesised that APOE $\varepsilon 2$ allele may exert a protection against Alzheimer's disease and that the APOE genotype specific effects on the disease may vary with age and sex. The purpose of this study was to examine these hypotheses by investigating the age or sex specific association between APOE genotype and incidence of Alzheimer's disease using the six years follow up data from the Kungsholmen project.

\section{METHODS}

\section{Study population}

The study population was derived from the Kungsholmen project, a community based cohort study of aging and dementia. Design of the project and inception of the cohort have been fully reported elsewhere. ${ }^{19} 20$ Briefly, the initial population of the project included all eligible inhabitants that were 75 years and older in October 1987 and were living in the Kungsholmen district of Stockholm, Sweden. By a two phase design, 1473 subjects were identified as being free of dementia at baseline (1987 to 1989). Of these subjects, 172 refused to undertake the first follow up contact (1991 to 1993) or had moved out of Stockholm before the contact. Information on APOE genotype was missing for 316 persons. Therefore, the population for these analyses consisted of 985 subjects who underwent at least the first follow up examination. Among the 689 subjects who were alive and remained non-demented at first follow up, 31 refused to participate in the second follow up evaluation (1994 to 1996). Medical records and death certificates were available for all the deceased subjects during the first $(n=145)$ and the 
second $(n=123)$ follow up periods. All parts of the project received approval from the ethics committee of Karolinska Institutet.

\section{Baseline data collection}

Data on age, sex, and cognitive function (assessed with the mini-mental state examination, MMSE) were collected following standard protocols. ${ }^{19} 20$ Educational level was measured by total years of formal schooling and divided into $<8$ years compared with $\geqslant 8$ years based on a previous study. ${ }^{21}$ Genomic DNA was extracted from peripheral blood samples that were taken during the baseline survey, and a standard polymerase chain reaction procedure was used for APOE genotyping. ${ }^{22}$ Blood pressure was measured by nurses on the right arm using a mercury sphygmomanometer. Information on history of heart disease (ICD-8 codes 410414,427 , and 428), cerebrovascular disease (ICD-8 codes 430-438), and diabetes mellitus (ICD-8 code 250) at baseline was derived from the computerised inpatient register system that covered all hospitals in the Stockholm area since 1969. This register system recorded up to six kinds of disorders that had been identified during each period of hospitalisation. Data on medical drug use were collected for the two weeks preceding the baseline survey. Antihypertensive drugs were defined as all medicines potentially used for lowering blood pressure (that is, the Anatomical Therapeutic Chemical classification system ${ }^{23}$ codes C02, C03, and C07).

\section{Diagnosis of incident Alzheimer's disease}

The incident cases were all people that developed Alzheimer's disease and were detected over the two follow up periods. The follow up evaluation and diagnostic procedure have been reported elsewhere. ${ }^{20}{ }^{24}$ In brief, all survivors underwent an extensive dementia examination at each follow up, including a structured interview by nurses, a comprehensive clinical examination by physicians, and neuropsychological assessments. The Diagnostic and Statistical Manual of Mental Disorders, revised third edition (DSM-III-R) criteria $^{25}$ were used to define dementia cases with a three step procedurethat is, two examining physicians independently made a preliminary diagnosis and a third opinion was asked in case of disagreement. The diagnosis of Alzheimer's disease required gradual onset, progressive deterioration, and lack of any other specific causes of dementia. Alzheimer's disease was diagnosed on a clinical basis, and neuropathological and imaging data were not available for the diagnosis. Our criteria for Alzheimer's disease were similar to those from the National Institute of Neurological and Communicative Disorders and Stroke-Alzheimer's Disease and Related Disorders Association for probable Alzheimer's disease. ${ }^{26}$ For deceased subjects, two physicians made the diagnosis through reviewing the medical records and death certificates.

\section{Data analysis}

The statistical differences were examined with $t$ test for continuous and $\chi^{2}$ test for categorical variables. We used logistic regression analysis to evaluate the effect of baseline characteristics on drop outs. Incidence rate was calculated as the number of cases divided by person years at risk of follow up. ${ }^{20}$ Cox proportional hazards models were constructed to estimate the relative risk of Alzheimer's disease, with corresponding 95\% confidence intervals (CI). We first examined the association between APOE genotype and incidence of Alzheimer's disease, with the $\varepsilon 3 / \varepsilon 3$ genotype as a reference category. The modifying effects of age and sex were then evaluated by stratified analysis. Finally, we examined the statistical interaction by including the two independent variables and their cross product term in the same model. Age (in years), sex, education, systolic pressure (indicator variables with categories of $<140,140$ to 159 , $\geqslant 160 \mathrm{~mm} \mathrm{Hg}$, and missing values), diastolic pressure (indicator variables with categories of $<70,70$ to 89 , $\geqslant 90 \mathrm{~mm} \mathrm{Hg}$, and missing values), vascular disease, baseline MMSE score, and antihypertensive drug use were considered as covariates in multivariate analyses. We estimated the potential contribution of APOE $\varepsilon 4$ allele to Alzheimer's disease by calculating the population attributable risk percentage $(\mathrm{PAR} \%),{ }^{27}$ that is $\mathrm{PAR} \%=\{p(r-1) /[1+p(r-1)]\} \times 100 \%$, where $p$ is the proportion of $\varepsilon 4$ allele in the population and $r$ is the relative risk estimated from a multiple model.

\section{RESULTS}

Of the initial dementia free cohort $(n=1473), 488$ subjects dropped out because of refusal of either the first follow up visit or the APOE genotyping. In a multiple logistic regression model, being a drop out was associated with older age (odds ratio per one year increment $1.10,95 \%$ CI 1.07 to 1.13 ) and a lower baseline MMSE score (odds ratio per one point increment $0.93,95 \%$ CI 0.89 to 0.97 ), but not significantly related to sex, educational level, vascular disease, or antihypertensive drug use.

The frequencies of different APOE genotypes among the 985 subjects were $0.3 \%(\mathrm{n}=3)$ for $\varepsilon 2 / \varepsilon 2,13.2 \%(\mathrm{n}=130)$ for $\varepsilon 2 / \varepsilon 3,1.9 \%(\mathrm{n}=19)$ for $\varepsilon 2 / \varepsilon 4,58.0 \%(\mathrm{n}=571)$ for $\varepsilon 3 / \varepsilon 3$, $24.3 \%(\mathrm{n}=239)$ for $\varepsilon 3 / \varepsilon 4$, and $2.3 \%(\mathrm{n}=23)$ for $\varepsilon 4 / \varepsilon 4$. Table 1 shows the baseline characteristics of the study participants by APOE genotypes. There was no significant difference in the distribution of these characteristics among APOE genotype groups.

\section{Relation of Alzheimer's disease to APOE genotypes}

During the total of 4754 person years of follow up (median 5.6, range 0.1 to 8.3 years), Alzheimer's disease was diagnosed in 206 subjects. Compared with APOE $\varepsilon 3 / \varepsilon 3$ genotype, the $\varepsilon 4$ allele was significantly associated with an increased risk of Alzheimer's disease in a dose-response fashion, whereas the $\varepsilon 2$ allele (that is, $\varepsilon 2 / \varepsilon 2$ or $\varepsilon 2 / \varepsilon 3$ genotype) was related to a reduced risk of the disease although it was not statistically significant (table 2 ). In this multiple Cox model, being female was associated with a relative risk of 1.7 (95\% CI 1.2 to 2.5$)$ for developing Alzheimer's disease.

\section{Relation of Alzheimer's disease to APOE genotypes by age and sex}

Overall, as shown in figure 1, the strength of association between APOE $\varepsilon 4$ allele and risk of Alzheimer's disease did not differ considerably by age group, but the point estimate of relative risk was more than doubled in men than in women. Conversely, the $\varepsilon 2$ related relative risk for Alzheimer's disease did not vary much by sex, whereas the $\varepsilon 2$ allele was significantly related to a reduced disease risk, especially in people under the age of 85 years. There was no protection of the $\varepsilon 2$ allele against Alzheimer's disease in the oldest old $(\geqslant 85$ years). Further analysis indicated that the $\varepsilon 2$ allele specific benefit was present mainly in women aged 75 to 84 years old $(\mathrm{n}=590,123$ Alzheimer cases; the multi-adjusted relative risk $0.4,95 \%$ CI 0.2 to 0.7 ). Among men who were 75 to 84 years of age $(n=208,26$ Alzheimer cases), no benefit from carrying the $\varepsilon 2$ allele was seen (multi-adjusted relative risk $1.6,95 \%$ CI 0.3 to 7.4 ). In the entire population, the multi-adjusted relative risks were 2.4 (95\% CI 1.0 to 6.0, $\mathrm{p}=0.06$ ) for the interaction term of $\varepsilon 2$ allele by age ( $\geqslant 85$ versus $<85$ years) and 0.4 (95\% CI 0.2 to 0.9 ) for the term of $\varepsilon 4$ allele by sex. No statistical interactions of the $\varepsilon 2$ allele with sex and of the $\varepsilon 4$ allele with age were detected. 
Table 1 Baseline characteristics of the study participants $(n=985)$ by the APOE genotypes

\begin{tabular}{|c|c|c|c|c|c|c|c|}
\hline \multirow[b]{2}{*}{ Characteristic } & \multicolumn{2}{|c|}{$\varepsilon 2 / \varepsilon 2$ or $\varepsilon 2 / \varepsilon 3(n=133)$} & \multicolumn{2}{|c|}{$\varepsilon 3 / \varepsilon 3(n=571)$} & \multicolumn{2}{|c|}{ any $\varepsilon 4(n=281)$} & \multirow[b]{2}{*}{$\mathrm{p}$ Value } \\
\hline & Number & $\%$ & Number & $\%$ & Number & $\%$ & \\
\hline \multicolumn{8}{|l|}{ Age group, years } \\
\hline 75 to 79 & 62 & 46.6 & 284 & 49.7 & 148 & 52.6 & \\
\hline 80 to 84 & 37 & 27.8 & 176 & 30.8 & 91 & 32.4 & \\
\hline 85 to 89 & 21 & 15.8 & 74 & 13.0 & 30 & 10.7 & \\
\hline$\geqslant 90$ & 13 & 9.8 & 37 & 6.5 & 12 & 4.3 & 0.26 \\
\hline Female sex & 102 & 76.7 & 420 & 73.6 & 216 & 76.9 & 0.51 \\
\hline Educational level $<8$ years & 80 & 60.2 & 329 & 57.6 & 157 & 55.9 & 0.71 \\
\hline MMSE* $^{*}$ score $<24$ & 6 & 4.5 & 25 & 4.4 & 16 & 5.7 & 0.69 \\
\hline Vascular disease $†$ & 27 & 20.3 & 127 & 22.2 & 49 & 17.4 & 0.26 \\
\hline Antihypertensive drug use & 59 & 44.4 & 246 & 43.1 & 127 & 45.2 & 0.84 \\
\hline \multicolumn{8}{|l|}{ Systolic pressuref, $\mathrm{mm} \mathrm{Hg}$} \\
\hline$<140$ & 20 & 15.4 & 95 & 17.1 & 50 & 17.9 & \\
\hline 140 to 159 & 57 & 43.8 & 184 & 33.0 & 101 & 36.2 & \\
\hline$\geqslant 160$ & 53 & 40.8 & 278 & 49.9 & 128 & 45.9 & 0.20 \\
\hline \multicolumn{8}{|l|}{ Diastolic pressure $\neq, \mathrm{mm} \mathrm{Hg}$} \\
\hline$<70$ & 10 & 7.7 & 49 & 8.8 & 31 & 11.1 & \\
\hline 70 to 89 & 87 & 66.9 & 339 & 60.9 & 174 & 62.4 & \\
\hline$\geqslant 90$ & 33 & 25.4 & 169 & 30.3 & 74 & 26.5 & 0.46 \\
\hline
\end{tabular}

\section{Population attributable risk estimation}

In comparison with the carriers of $\varepsilon 3 / \varepsilon 3$ genotype, the percentages of Alzheimer cases attributable to possession of the $\varepsilon 4$ allele were $14.6 \%$ in the entire population, $33.3 \%$ in men, and $10.5 \%$ in women.

\section{Additional analyses}

We repeated the analysis by using non- $\varepsilon 4$ allele as a reference group. The adjusted relative risk of Alzheimer's disease related to any $\varepsilon 4$ allele was 1.6 (95\% CI 1.2 to 2.2 ), with the relative risk of 1.5 (95\% CI 1.1 to 2.1 ) for one $\varepsilon 4$ allele and 3.2 (95\% CI 1.7 to 6.2 ) for two copies of the allele ( $\mathrm{p}$ for trend $<0.01)$. The $\varepsilon 4$ allele related risk for Alzheimer's disease was doubled in men (relative risk $3.1,95 \%$ CI 1.4 to 6.5 ) than in women (relative risk $1.5,95 \%$ CI 1.0 to $2.0, p=0.02$ ), with the relative risk of 0.4 (95\% CI 0.2 to 0.9 ) for the interaction term of any $\varepsilon 4$ by sex. There was no statistical interaction of $\varepsilon 4$ allele with age. Furthermore, to assess the potential influence of APOE genotype related selective survivals, we reanalysed the data by either additionally adjusting for the vital status at the time of Alzheimer's disease being diagnosed or excluding the deceased subjects from the study population, which yielded results similar to those from the initial analysis (data not shown).

\section{DISCUSSION}

In this comparatively homogenetic population of community dwelling people aged $\geqslant 75$ years, we found: (1) the $\varepsilon 2$ allele confers a protection against Alzheimer's disease in the younger old people ( $<85$ years); and (2) the strength of association between APOE $\varepsilon 4$ allele and risk of Alzheimer's disease is stronger in men than in women. It seems unlikely that the potential confounding biases or selective survivals could explain the observed age and sex difference in the APOE genotype specific effects on the disease.

Numerous studies have reported that the APOE $\varepsilon 4$ allele specific risk for Alzheimer's disease increases with age until around 70 and declines thereafter. ${ }^{5}{ }^{13-15}$ We were not able to evaluate the age variation of the $\varepsilon 4$ allele-Alzheimer association before 75 years, but our data showed that the risk effect of the $\varepsilon 4$ allele on Alzheimer's disease might not vary by age after 75 . This notion is in line with a previous population study, ${ }^{10}$ in which the $\varepsilon 4$ related risk for Alzheimer's disease appeared to have no pronounced variation with age after 75 years. A few population based studies have shown a stronger female specific impact of the $\varepsilon 4$ allele on Alzheimer's disease or on the development of cognitive decline ${ }^{28-30}$ This finding has been viewed as a partial support for the higher incidence of Alzheimer's disease in women than in men. ${ }^{29}{ }^{30}$ By contrast, the recent population based study from north Europe indicated a stronger association between the APOE $\varepsilon 4$ allele and Alzheimer's disease in men than in women. ${ }^{16}{ }^{17}$ In accordance with this recent study, we found an interaction between APOE $\varepsilon 4$ and sex on the occurrence of Alzheimer's disease, in which the $\varepsilon 4$ allele exerted a higher risk to develop Alzheimer's disease in men compared with women. As no sex difference in the $\varepsilon 4$ allele frequency was seen in our population, the substantial population attributable risk of Alzheimer's disease, which resulted from possession of the $\varepsilon 4$ allele in men $(33.3 \%)$ compared with women $(10.5 \%)$, is due primarily to a stronger APOE $\varepsilon 4$ allele-Alzheimer's disease association in men.

Few population based prospective studies have so far assessed the gene-dose effect of the $\varepsilon 4$ allele on the risk of

Table 2 Crude incidence rates (per 1000 person years) and adjusted relative risks (95\% Cls) of Alzheimer's disease by APOE genotypes

\begin{tabular}{llllll}
\hline APOE genotype & Number of subjects & Number of cases & Incidence rate & Model $\mathbf{1}^{*}$ & ${\text { Model } \mathbf{2}^{*}}$ \\
\hline$\varepsilon 3 / \varepsilon 3$ & 571 & 110 & 40.5 & 1.0 (reference) & $1.0($ reference) \\
$\varepsilon 2 / \varepsilon 2$ or $\varepsilon 2 / \varepsilon 3$ & 133 & 24 & 37.5 & $0.8(0.5$ to 1.2$)$ & $0.8(0.5$ to 1.2$)$ \\
$\varepsilon 2 / \varepsilon 4$ or $\varepsilon 3 / \varepsilon 4$ & 258 & 61 & 54.7 & $1.4(1.1$ to 2.0$)$ & $1.4(1.0$ to 2.0$) \dagger$ \\
$\varepsilon 4 / \varepsilon 4$ & 23 & 11 & 132.1 & $3.9(2.1$ to 7.3$)$ & $3.1(1.6$ to 5.9$)$ \\
\hline
\end{tabular}

*The relative risks $(95 \% \mathrm{Cls})$ were derived from different Cox proportional hazards models: model 1 included age, sex, education, and APOE genotype; model 2 included all variables in model 1 plus baseline MMSE score, systolic pressure, diastolic pressure, vascular disease, and the use of antihypertensive drugs; $\mathrm{tp}=0.03$. 

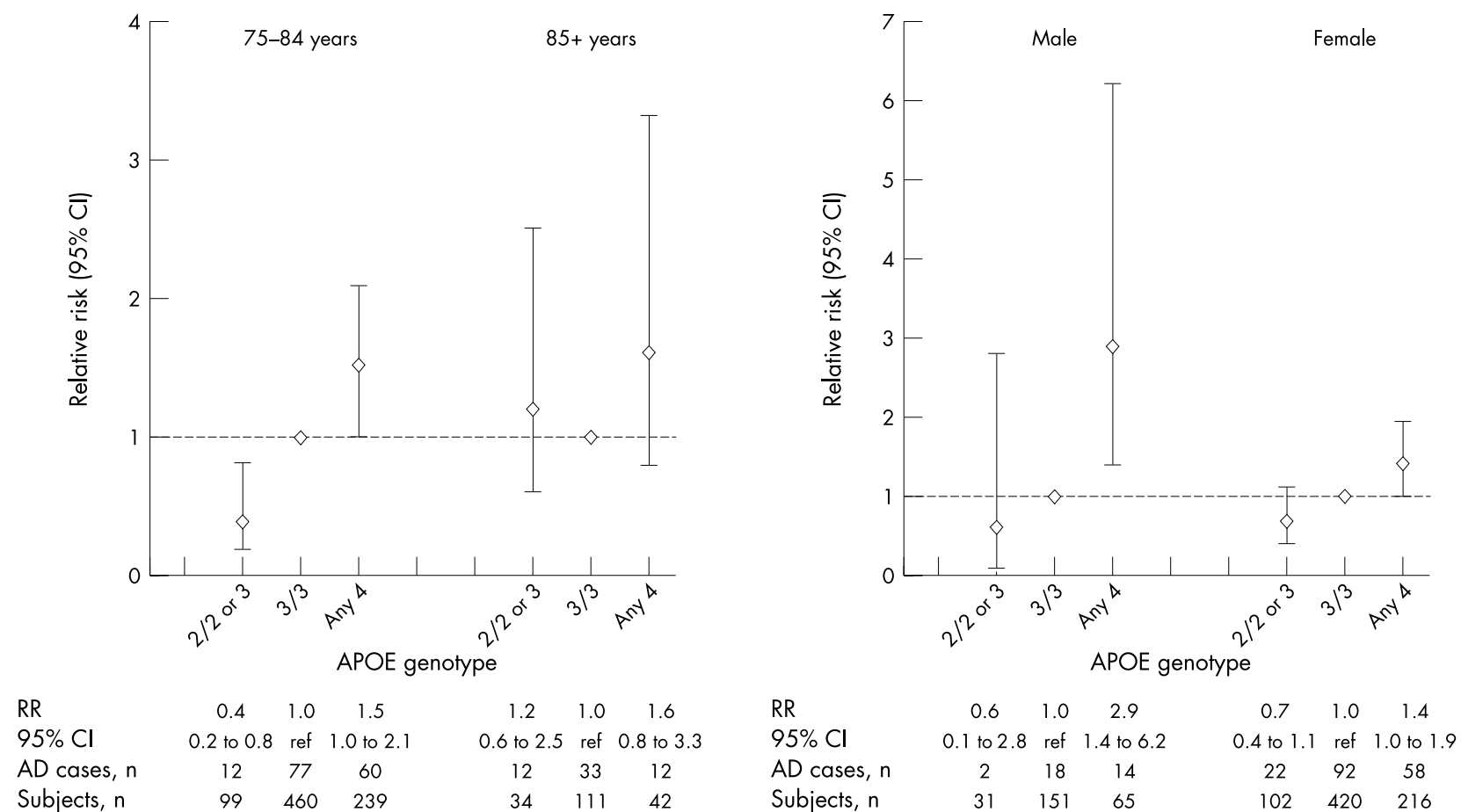

Figure 1 Relation of Alzheimer's disease (AD) to APOE genotype by age group (left) and sex (right). The relative risk (RR) and $95 \%$ confidence interval (CI) were estimated with adjustment for age (in years), education, baseline MMSE score, systolic pressure, diastolic pressure, vascular disease, antihypertensive drug use, and if applicable, for sex.

Alzheimer's disease, partly because of limited number of APOE $\varepsilon 4$ homozygous people in the general populations. In the nested case-control analysis of the Rotterdam study, ${ }^{10}$ the risk ratios of Alzheimer's disease were 1.8 for the $\varepsilon 3 / \varepsilon 4$ genotype and 6.2 for the homozygous $\varepsilon 4$ allele compared with the $\varepsilon 3 / \varepsilon 3$ genotype, while in the Copenhagen heart study the corresponding figures were 3.3 and 10.1. ${ }^{8}$ Our study showed a clear dose-response relation between the number of $\varepsilon 4$ alleles and the risk of Alzheimer's disease, but the strength of the dose association was less stronger than those previously reported. Differences in characteristics of the study population as well as in the consideration of potential confounders may largely explain these discrepancies. For instance, our study population was much older and more covariates were taken into account in our analysis. In addition, some studies have estimated the $\varepsilon 4$ allele related risk for Alzheimer's disease by using non- $\varepsilon 4$ allele as a referent category. ${ }^{31}{ }^{32}$ To compare with these studies, we also estimated the risk effect of the $\varepsilon 4$ allele versus non- $\varepsilon 4$ allele in the additional analysis, but this may generally lead to an overestimation of the risk role of the allele because of the potential protective effect of the $\varepsilon 2$ allele on the development of Alzheimer's disease.

A meta-analysis study suggested that the $\varepsilon 2 / \varepsilon 3$ genotype (probably the $\varepsilon 2 / \varepsilon 2$ genotype) could exert protection against Alzheimer's disease, but it did not vary with age. ${ }^{5}$ Recently, the association between $\varepsilon 2$ allele and a decreased risk of Alzheimer's disease has been reported in a few population based studies..$^{9-12}$ The non-significantly protective effect of the $\varepsilon 2$ allele seemed similar across age strata in the Rotterdam cohort aged 55 years and older. ${ }^{10}$ In the MRC cognitive function and ageing study, ${ }_{11}^{11}$ subjects possessing the $\varepsilon 2$ allele were found to have a lower risk of dementia, but the decreased risk was not significant because of small numbers. Sex specific analysis in this study did not show any difference in the allele effect between men and women. The cohort study of elderly Catholic clerics in the United States showed that APOE $\varepsilon 2$ allele could protect against episodic memory decline, a defining feature of Alzheimer's disease. ${ }^{12}$ The protection of the $\varepsilon 2$ allele on Alzheimer's disease was less evident, largely because of limited statistical power. On the other hand, two population based studies showed no evidence of any protection of the $\varepsilon 2$ allele against Alzheimer's disease. ${ }^{68}$ One of the important findings in our study was that the benefit of $\varepsilon 2$ allele, mostly the $\varepsilon 2 / \varepsilon 3$ genotype, on Alzheimer's disease was age dependent-that is, the apparent protection existed mainly in people aged 75 to 84 years. Although further analysis showed that the protection of $\varepsilon 2$ allele against Alzheimer's disease was present only among women, we were not able to determine whether there was a real sex difference in the effect of $\varepsilon 2$ allele in this age group because our study cohort included too few male carriers of the allele and the confidence intervals were largely overlapping. Further clarification of the age and sex differences in the $\varepsilon 2$ allele effect on Alzheimer's disease may be helpful to understand the previous inconsistent findings. For instance, the $\varepsilon 2$ allele specific effect for Alzheimer's disease reported in some studies might not be specified by age and sex.

Neuropathological studies have linked the risk and benefit effects of different APOE genotypes to the densities of $\beta$ amyloid protein and neurofibrillar tangles in the brain, ${ }^{33}{ }^{34}$ which are the hallmark features of Alzheimer lesions. What are the possible explanations for the age and sex variations of the APOE genotype specific effects on the disease? Firstly, in our cohort, more men were affected by vascular disease $(24.7 \%$ versus $19.2 \%, p=0.07)$, whereas more women were treated with antihypertensive drugs $(46.7 \%$ versus $35.2 \%$, $\mathrm{p}<0.01$ ). This may partially explain the sex difference in the $\varepsilon 4$ allele-Alzheimer association because previous studies ${ }^{2} 3536$ have shown that vascular disorders may advance the dementia process, while the use of antihypertensive drugs may diminish the risk effect of $\varepsilon 4$ allele on the disease. Secondly, the age and sex differences in the $\varepsilon 4$ specific risk may reflect the variant severities of Alzheimer lesions in the 
brain, as a neuropathological study indicated that the $\varepsilon 4$ allele was related to senile plaques across all ages for men, whereas for women such a relation existed only in those under 80 years. ${ }^{37}$ Thirdly, previous studies have revealed a sex-differential effect of APOE genotype on plasma lipids metabolism and atherosclerotic disorders. ${ }^{38}{ }^{39}$ In the Framingham study, for instance, after controlling for major potential confounders, possession of the $\varepsilon 4$ allele (compared with $\varepsilon 3 / \varepsilon 3$ ) was associated with a greater risk for cardiovascular disease only in men. As serum cholesterol and atherosclerosis may act directly or in combination with APOE $\varepsilon 4$ to increase the risk of Alzheimer's disease, ${ }^{20}$ it could contribute to the sex difference in the $\varepsilon 4$ alleleAlzheimer association. The age and sex differential $\varepsilon 2$ alleleAlzheimer's disease association needs to be verified before any explanations can be offered.

A potential limitation of this study is that information on APOE genotypes was missing for nearly one third of the initial eligible dementia free subjects. However, drop out analysis suggested that this might not seriously harm the representativeness of our population, and sensitivity analysis showed no evidence for the potential influence of missing values on the estimate of APOE genotype-dementia association. ${ }^{35}$ Furthermore, the lack of neuroimaging and necropsy verified information for the diagnosis of Alzheimer's disease may lead to misclassification, but this may merely attenuate the association between APOE genotypes and risk of the disease. Furthermore, the study population consisted of persons with a minimum age of 75 years at entry. Thus, it may not be justified in generalising these findings to younger people. Finally, the multiple hypothesis tests within a single dataset may lead to a higher rate of type I error (that is, false positive inference), which emphasises the need for further confirmation of our findings.

In summary, the APOE $\varepsilon 4$ allele has a gene-dose effect on the risk of Alzheimer's disease, whereas the $\varepsilon 2$ allele confers a protection against the disease. The APOE genotype specific risks for Alzheimer's disease vary by age and sex. These findings, if replicated, may have relevant indications for genetic counsel (for example, age and sex should be specified to estimate the APOE genotype related risk) as well as for the aetiology of Alzheimer's disease.

\section{ACKNOWLEDGEMENTS}

We thank the members in the Kungsholmen project study group for their cooperation in data collection and management.

\section{Authors' affiliations}

C Qiu, M Kivipelto, H Agüero-Torres, B Winblad, L Fratiglioni, Ageing Research Centre, Division of Geriatric Epidemiology and Medicine, Department of Neurotec, Karolinska Institutet and the Stockholm Gerontology Research Centre, Stockholm, Sweden

Funding: research grants were received from the Swedish Research Council, the Karolinska Institutet, the Gun and Bertil Stohne Foundation, the Gamla Tiänarinnor Foundation, and the Swedish Alzheimer Foundation.

Competing interests: none declared.

\section{REFERENCES}

1 National Institute on Aging/Alzheimer's Association Working Group. Apolipoprotein E genotyping in Alzheimer's disease. Lancet 1996:347:1091-5.

2 Kivipelto M, Helkala E-L, Laakso MP, et al. Apolipoprotein E $\varepsilon 4$ allele, elevated midlife total cholesterol level, and high midlife systolic pressure are independent risk factors for late-life Alzheimer disease. Ann Intern Med 2002;137:149-55.

3 Nussbaum RL, Ellis CE. Alzheimer's disease and Parkinson's disease. N Engl J Med 2003;348:1356-64

4 van Duijn CM, De Knijff $P$, Wehnert A, et al. The apolipoprotein E $\varepsilon 2$ allele is associated with an increased risk of early-onset Alzheimer's disease and a reduced survival. Ann Neurol 1995;37:605-10.
5 Farrer LA, Cupples LA, Haines JL, et al. Effects of age, sex, and ethnicity on the association between apolipoprotein $\mathrm{E}$ genotype and Alzheimer disease: a meta-analysis. JAMA 1997;278:1349-56.

6 Katzman R, Zhang M-Y, Chen PJ, et al. Effects of apolipoprotein E on dementia and aging in the Shanghai survey of dementia. Neurology 1997;49:779-85.

7 Ganguli M, Chaandra V, Kamboh MI, et al. Apoplipoprotein E polymophism and Alzheimer disease: the Indo-US cross-national dementia study. Arch Neurol 2000;57:824-30.

8 Frikke-Schmidt R, Nordestgaard BG, Thudium D, et al. APOE genotype predicts $A D$ and other dementia but not ischemic cerebrovascular disease. Neurology 2001;56:194-200.

9 Meyer MR, Tschanz JT, Norton MC, et al. APOE genotype predicts when-not whether-one is predisposed to develop Alzheimer disease. Nat Genet 1998; 19:321-2.

10 Slooter AJ, Cruts M, Kalmiin S, et al. Risk estimates of dementia by apolipoprotein $\mathrm{E}$ genotype from a population-based incidence study: the Rotterdam study. Arch Neurol 1998;55:964-8.

11 Yip AG, Brayne C, Easton D, et al. Apolipoprotein E4 is only a weak predictor of dementia and cognitive decline in the general population. J Med Genet 2002;39:639-43.

12 Wilson RS, Bienias JL, Berry-Kravis E, et al. The apolipoprotein E $\varepsilon 2$ allele and decline in episodic memory. J Neurol Neurosurg Psychiatry 2002:73:672-7.

13 Tang $M-X$, Stern $Y$, Marder K, et al. The APOE- $\varepsilon 4$ allele and the risk of Alzheimer disease among African Americans, whites, and Hispanics. JAMA 1998;279:751-5.

14 Juva K, Verkkoniemi A, Viramo $P$, et al. APOE \&4 does not predict mortality, cognitive decline, or dementia in the oldest old. Neurology 2000;54:412-15

15 Miech RA, Breitner JCS, Zandi PP, et al. Incidence of AD may decline in the early 90 s for men, later for women: the Cache County study. Neurology 2002;58:209-18.

16 Kivipelto M, Helkala E-L, Nissinen A, et al. Vascular risk factors, ApoE \&4 allele, and gender and the risk of Alzheimer's disease: perspectives on prevention. Drug Dev Res 2002;56:85-94.

17 Anttila T, Helkala E-L, Kivipelto M, et al. Midlife income, occupation, APOE status, and dementia: a population-based study. Neurology 2002;59:887-93

18 Molero AE, Pino-Ramírez G, Maestre GE. Modulation by age and gender of risk for Alzheimer disease and vascular dementia associated with the apolipoprotein E- $\varepsilon 4$ allele in Latin Americans: findings from the Maracaibo aging study. Neurosci Lett 2001;307:5-8.

19 Fratiglioni L, Viitanen M, Bäckman L, et al. Occurrence of dementia in advanced age: the study design of the Kungsholmen project. Neuroepidemiology 1992;11(suppl 1):29-36.

20 Fratiglioni $L$, Viitanen $M$, von Strauss $E$, et al. Very old women at highest risk of dementia and Alzheimer's disease: incidence data from the Kungsholmen project, Stockholm. Neurology 1997;48:132-8.

21 Qiu CX, Bäckman L, Winblad B, et al. The influence of education on clinically diagnosed dementia: incidence and mortality data from the Kungsholmen project. Arch Neurol 2001;58:2034-9.

22 Basun H, Corder EH, Guo Z, et al. Apolipoprotein E polymorphism and stroke in a population sample aged 75 years or more. Stroke 1996;27:1310-15.

23 Nordic Council on Medicines. Guidelines for ATC Classification. Uppsala, Sweden: Nordic Council on Medicines, 1985 (NLN Publication no 16).

24 Fratiglioni L, Grut M, Forsell Y, et al. Clinical diagnosis of Alzheimer's disease and other dementias in a population survey: agreement and causes of disagreement in applying Diagnostic and Statistical Manual of Mental Disorders, Revised Third Edition, criteria. Arch Neurol 1992;49:927-32.

25 American Psychiatric Association. Diagnostic and statistical manual of mental disorders, revised third edition (DSM-III-R). Washington, DC: American Psychiatric Association, 1987

26 McKhann G, Drachman D, Folstein M, et al. Clinical diagnosis of Alzheimer's disease: report of the NINCDS-ADRDA Work Group under the auspices of Department of Health and Human Service Task Force on Alzheimer's Disease. Neurology 1984;34:939-44.

27 Kelsey JL, Thompson WD, Evans AS. Methods in observational epidemiology. New York: Oxford University Press, 1986

28 Hyman BT, Gomez-Isla T, Briggs M, et al. Apolipoprotein E and cognitive change in an elderly population. Ann Neurol 1996;40:55-66.

29 Payami H, Zareparsi S, Montee KR, et al. Gender difference in apolipoprotein E-associated risk for familial Alzheimer disease: a possible clue to the higher incidence of Alzheimer disease in women. Am J Hum Genet 1996;58:803-11.

30 Mortensen EL, Hogh P. A gender difference in the association between APOE genotype and age-related cognitive decline. Neurology 2001:57:89-95.

31 Kukull WA, Higdon R, Bowen JD, et al. Dementia and Alzheimer disease incidence: a prospective cohort study. Arch Neurol 2002;59:1737-46.

32 Evans DA, Bennett DA, Wilson RS, et al. Incidence of Alzheimer disease in a biracial urban community: relation to apolipoprotein E allele status. Arch Neurol 2003:60:185-9.

33 Polvikoski T, Sulkava R, Hattia M, et al. Apolipoprotein E, dementia, and cortical deposition of $\beta$-amyloid protein. N Engl J Med 1995;333:1242-7.

34 Lippa CF, Smith TW, Saunders AM, et al. Apolipoprotein E- $\varepsilon 2$ and Alzheimer's disease: genotype influences pathologic phenotype. Neurology 1997:48:515-19. 
35 Zhu L, Fratiglioni L, Guo Z, et al. Incidence of dementia in relation to stroke and the apolipoprotein $\mathrm{E} \varepsilon 4$ allele in the very old: findings from a populationbased longitudinal study. Stroke 2000;31:53-60.

36 Guo Z, Fratiglioni L, Viitanen M, et al. Apolipoprotein E genotypes and incidence of Alzheimer's disease among persons aged 75 years and older: variation by use of antihypertensive medication? Am J Epidemiol variation by use of

37 Ghebremedhin E, Schultz C, Thal DR, et al. Gender and age modify the association between $A P O E$ and $A D$-related neuropathology. Neurology 2001;56:1696-701.
38 Reilly SL, Ferrell RE, Kottke BA, et al. The gender-specific apolipoprotein E genotype influence on the distribution of plasma lipids and apolipoproteins in the population of Rochester, Minnesota: regression relationships with concomitants. Am J Hum Genet 1992;51:1311-24.

39 Lahoz C, Schaefer EJ, Cupples LA, et al. Apolipoprotein E genotype and cardiovascular disease in the Framingham heart study. Atherosclerosis $2001 ; 154: 529-37$

40 Hofman A, OH A, Breteler MB, et al. Atherosclerosis, apolipoprotein E, and prevalence of dementia and Alzheimer's disease in the Rotterdam study. Lancet 1997;349:151-4.

\section{NEUROLOGICAL PICTURE}

Neurosyphilis with complex partial status epilepticus and mesiotemporal MRI abnormalities mimicking herpes simplex encephalitis

A 48 year old man suffered a $9 \mathrm{~h}$ dreamy state with automatisms. The neurological examination revealed disinhibited behaviour, a positive Romberg sign, and absent abdominal and weak tendon reflexes. Neuropsychological examination showed a selective reduction of short term spatial memory. A brain MRI performed after 2 weeks showed a prevalently cortical lesion in the right temporal and basal frontal lobes, which was slight hypointense in $\mathrm{Tl}$ weighted images and hyperintense in DP and T2 weighted images (fig 1), without enhancement. Carbamazepine was introduced. About a month later a second brain MRI revealed that the lesion was reduced to a small area in the right mesiotemporal region (fig 2), hypointense in $\mathrm{Tl}$ weighted and hyperintense in $\mathrm{T} 2$ weighted images, without enhancement after intravenous gadolinium; the right temporal horn was greater than the controlateral. CSF analysis showed normal glucose content, 12 lymphocytes $/ \mathrm{mm}^{3}$, slight elevated protein level $(63 \mathrm{mg} / \mathrm{dL}$, normal range: $20-40)$, a positive Link's index $(3.5$, normal $<0.75)$, 5 IgG oligoclonal bands, a positive VDRL test, a negative microscopic examination for mycobacteria and cryptococcus, and a negative PCR test for herpes simplex virus (HSV) 1 and HSV 2 DNA. Serum VDRL and TPHA were both positive. Serum antibodies to Borrelia burgdorferi, Toxoplasma gondii, and HIV 1 and HIV 2 were negative. The patient received intravenous penicillin $G$ at a dosage of 4 million IU four times a day for 10 days.

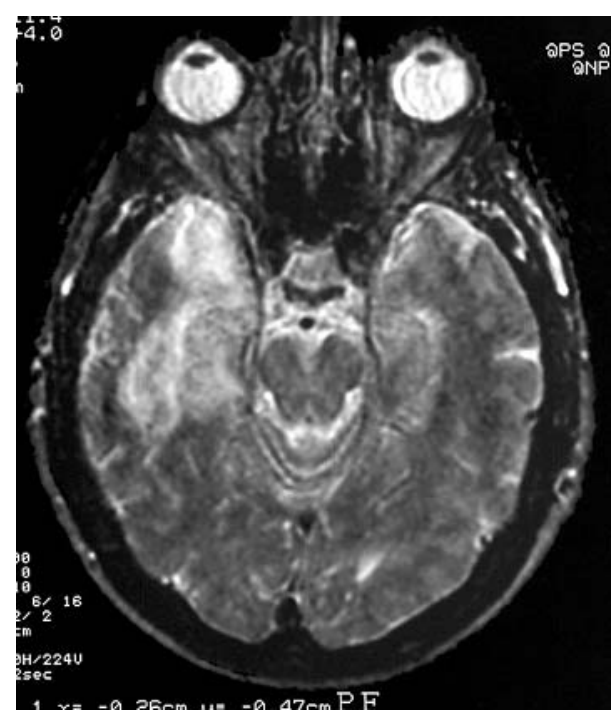

Figure 1 MRI shows an area of high signal on T2 weighted image in right temporal lobe.

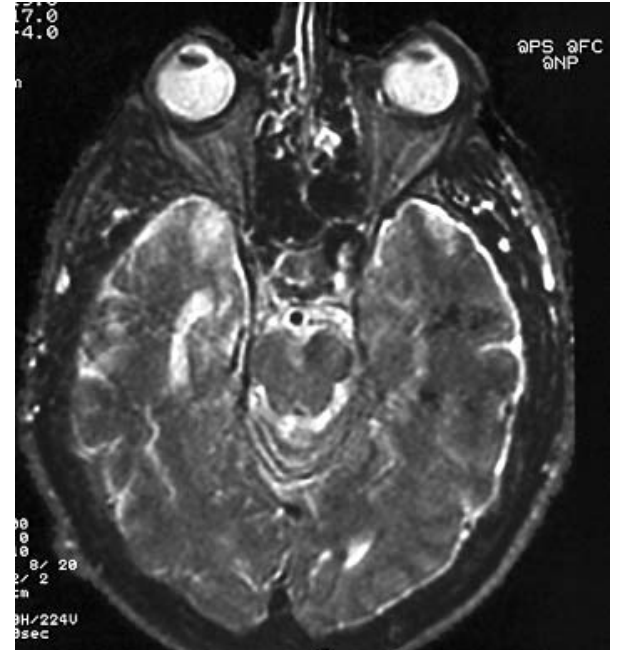

Figure 2 MRI shows a small area of high signal in temporal lobe with atrophic dilatation of temporal horn.

Complex partial status epilepticus (CPSE) has been observed with neurosyphilis. ${ }^{1}$ The pathogenesis is thought to be the mesiotemporal localisation of luetic vasculitis. The MRI shows cortical and subcortical hyperintensity in T2 weighted images, probably due to both cytotoxic and vasogenic oedema. ${ }^{2}$ The images are similar to those of HSV encephalitis. $^{3}$ The T2 hyperintense lesion in the right temporal lobe and fronto-basal region only evident on the first MRI examination and mimicking HSV encephalitis may be due to transient oedema caused by the $\mathrm{CPSE}^{4}$ and spontaneously resolved before penicillin treatment. The small right mesiotemporal lesion evident on the second MRI examination may have a vasculitic origin and it may be responsible for CPSE. We recommend short term repetition of brain MRI and CSF examination for differential diagnosis of mesiotemporal syndromes.

E Marano, F Briganti, F Tortora, A Elefante, A De Rosa, F Maiuri, A Filla Department of Neurological Sciences, Federico II University of Naples, S Pansini, 580131 Naples, Italy; eirtm@tin.it

\section{References}

1 Heald A, Connolly S, Hudgson P. Neurosyphilis presenting as complex partial status epilepticus. Eur Neurol 1996;36:111-2.

2 Brightbill TC, Ihmeidan IH, Post MJ, et al. Neurosyphilis in HIV-positive and HIV-negative patients: neuroimaging findings. Am J Neuroradiol 1995; 16:703-11.

3 Bash S, Hathout GM, Cohen S. Mesiotemporal T2-weighted hyperintensity: neurosyphilis mimicking herpes encephalitis. Am J Neuroradiol 2001;22(2):314-6.

4 Amato C, Elia M, Musumeci SA, et al. Transient MRI abnormalities associated with partial status epilepticus: a case report. Eur J Radiol 2001;38:50-54. 